\title{
VALIDACIÓN DE UN INSTRUMENTO PARA MEDIR EL NIVEL DE SATISFACCIÓN DE PACIENTES HOSPITALIZADOS CON RESPECTO AL SERVICIO DE NUTRICIÓN
}

\author{
VALIDATION OF AN INSTRUMENT TO MEASURE THE SATISFACTION LEVEL OF HOSPITALIZED PATIENTS WITH \\ RESPECT TO THE NUTRITION SERVICE \\ Elizabeth Rojas-Apolinario', Luis Roldan-Arbieto2,3, Jhony A. De La Cruz-Vargas ${ }^{4}$
}

\begin{abstract}
RESUMEN
Objetivo: Evaluar la validez y fiabilidad de un instrumento adaptado a partir de la escala SERVPERF para medir el nivel de satisfacción de pacientes con respecto al Servicio de Nutrición durante su hospitalización en una Institución de Salud en Lima, Perú. Métodos: Estudio transversal, descriptivo con componentes analíticos de validación del cuestionario SERVPERF adaptado para pacientes hospitalizados que reciben el Servicio de Nutrición. Se realizó una prueba piloto con posterior análisis de propiedades psicométricas de validez y fiabilidad mediante análisis factorial exploratorio de componentes principales y cálculo de alfa de Cronbach. Resultados: Se reporta una muestra de 100 pacientes, atendidos en la Clínica Good Hope, en el período de enero a mayo del año 2018. La prueba de adecuación de Kaiser-Meyer Olkin fue de 0.778 y la prueba de esfericidad de Barlett de 607.4 ( $p<0.000$ ). La varianza acumulada explicada por los 5 factores fue de $69.712 \%$. La fiabilidad mostró un alfa de Cronbach de 0.848 . Se clasificaron 3 factores con 15 ítems, redefiniéndose un factor como "Experiencia gastronómica". Conclusión: El instrumento adaptado y validado SERVNUT posee suficientes propiedades psicométricas para ser considerado una herramienta útil y fiable para medir la satisfacción de pacientes hospitalizados que reciben el Servicio de Nutrición en instituciones privadas de salud y con potencial aplicación usuarios en los diversos sectores de salud del Perú.
\end{abstract}

Palabras clave: Validación; Satisfacción; Nutrición; SERVPERF. (fuente: DeCS BIREME)

\begin{abstract}
Objetive: To evaluate the validity and reliability of an instrument adapted from the SERVPERF scale to measure the level of satisfaction Patients with respect to the Nutrition Service during their hospitalization in a Health Institution in Lima, Peru. Methods: Cross-sectional, descriptive study with analytical components validating the SERVPERF questionnaire adapted for hospitalized patinetes receiving the Nutrition Service. A pilot test was carried out with subsequent analysis of psychometric properties of validity and reliability by means of exploratory factorial analysis of main components and calculation of Cronbach's alpha. Results: A sample of 100 patinetas, seen at the Good Hope Clinic, was reportead from January to May of the year 2018. The Kaiser-Meyer Olkin adequacy test was 0.778 and the Barlett's sphericity test was $607.4(p<0.000)$. The cumulative variance explained by the 5 factors was $69,712 \%$. The reliability showed a Cronbach's alpha of 0.848 . Three factors were classified with 15 items, redefining a factor such as "Gastronomic experience". Conclusion: The adapted and validated instrument SERVNUT has enough psychometric properties to be considered a useful and reliable tool to measure the satisfaction of hospitalized patients receiving the Nutrition Service in private health institutions and with potential user application in the different sectors of health of Peru.
\end{abstract}

Key words: Validation; Satisfaction; Nutrition; SERVPERF. (source: MeSH NLM)

\footnotetext{
1 Departamento de Nutrición y Dietética, Clínica Good Hope, Lima-Perú.

${ }^{2}$ Unidad de Bioestadística, INICIB. FAMURP, Lima-Perú.

${ }^{3}$ Escuela de Post-Grado de la Universidad San Ignacio de Loyola, Lima-Perú.

${ }^{4}$ Instituto de Investigación en Ciencias Biomédicas (INICIB), Facultad de Medicina Humana, Universidad Ricardo Palma (FAMURP), Lima-Perú.
}

Citar como: Elizabeth Rojas-Apolinario, Luis Roldan-Arbieto, Jhony A. De La Cruz-Vargas. Validación de un instrumento para medir el nivel de satisfacción de pacientes hospitalizados con respecto al servicio de nutrición. [Artículo Original].2018;18(4):65-73. (Octubre 2018). DOl 10.25176/RFMH.v18.n4.1733 


\section{INTRODUCCIÓN}

En la actualidad la"Calidad del servicio"se ha convertido en un área transcendental de estudio, debido a su impacto en el desempeño de las organizaciones, reducción de los costos, lealtad de los usuarios y la rentabilidad'.

Importantes modelos de Gestión dela Calidad como ISO (InternationalOrganizationforStandardization) ${ }^{2}$,EFQM (European Foundation for Quality Management) (3,4 $^{3,4}$ o los criterios de acreditación aplicados a niveles asistenciales de la Joint Commission ${ }^{5}$, han sentado las bases para medir la calidad de los Servicios con sus normativas; sin embargo autores como Donabedian6 y Ware ${ }^{7}$ defienden la idea de que los mismos usuarios contribuirían en la evaluación de la calidad y proporcionarían desde su perspectiva, una valoración más cercana del servicio recibido lo cual ayudaría a los directivos en la toma de posteriores decisiones en bien de las organizaciones. Es por ello en la literatura se cuenta con instrumentos de fácil aplicación como encuestas y cuestionarios que evalúan el nivel de satisfacción alcanzado tras el servicio brindado ${ }^{8}$.

Parasuraman et $\mathrm{al}^{9}$, desarrollaron una escala de medición de la satisfacción de los usuarios, que es considerada una de las mayores contribuciones a la medición de la calidad de atención en las organizaciones que brindan servicios, incluyendo los de salud, sin embargo Cronin y Taylor ${ }^{10,11}$ llegaron a la conclusión que dicho modelo, parece no ser el más adecuado al momento de evaluar la calidad del servicio y elaboraron el modelo SERVPERF, cuyo nombre es debido a la exclusiva atención que presta a la valoración del desempeño (SERVice PERFormance) para medir la calidad de servicio. El razonamiento que sustenta el SERVPERF está relacionado con los problemas de interpretación del concepto de expectativa en la variabilidad del transcurso de la prestación del servicio y en la redundancia en relación a las percepciones del servicio recibido. Estructuralmente tiene los mismos ítems y dimensiones que SERVQUAL teniendo como única diferencia la eliminación de medición de las expectativas, haciendo el instrumento más práctico a la hora del recojo de los datos.

En los Servicios de Salud un aspecto importante que influye en la satisfacción de los pacientes hospitalizados es la nutrición que reciben ${ }^{12,13}$. Los Servicios de Nutrición que no solo cumplen sino que superan las expectativas del paciente tendrán gran impacto en la satisfacción general de la Institución ${ }^{14}$. Aunado a ello, el papel de los Servicios de Nutrición se ha vuelto cada vez más importante debido a los problemas asociados con la desnutrición ${ }^{15,16}$. Sin embargo en los últimos tiempos, ha habido una tendencia a utilizar herramientas validadas que incluyen preguntas sobre los servicios de alimentos en general, pero no están diseñadas para centrarse totalmente en el servicio de nutrición hospitalario y medir específicamente el nivel de satisfacción de sus usuarios durante el tiempo de hospitalización ${ }^{17}$.

Es por ello que el objetivo de este estudio fue desarrollar un cuestionario válido y confiable específico para el Servicio de Nutrición adaptado a nuestro medio considerando las dimensiones del modelo SERVPERF.

\section{MÉTODOS}

\section{Diseño:}

Planteamos un estudio transversal, descriptivo con componentes analíticos de adaptación y validación de la encuesta "SERVPERF" para medir el nivel de satisfacción de los pacientes hospitalizados con respecto al Servicio de Nutrición.

\section{Sujetos:}

La población objetivo estaba conformada por pacientes hospitalizados en la Clínica Good Hope cuyas edades oscilan entre 18 a 59 años y que recibían dieta blanda sin mayores restricciones alimenticias por su estado de salud.

La muestra fue calculada con un nivel de confianza del $95 \%$, un nivel de precisión del 7\%, una tasa de no respuesta de un 5\% y una tasa de satisfacción del $77 \%$ obteniéndose un tamaño de muestra de 100 pacientes. La toma de muestra se realizó por el método sistemático, por cada 5 pacientes que ingresaban y cumplían con los criterios mencionados eran seleccionados para la toma de datos. El estudio se realizó durante los meses de enero a mayo con la autorización previa del Comité de docencia de la Clínica Good Hope.

\section{Adaptación del cuestionario:}

La primera versión SERVNUT (Satisfacción de los usuarios del Servicio de Nutrición) 1.0 diseñado en este estudio se basa en el modelo SERVPERF, midiendo la satisfacción utilizando tan solo la percepción, a través de 15 ítems agrupados en las 5 dimensiones utilizadas por el modelo SERVPERF, haciendo uso de la escala de respuestas elementos de tipo Likert de 5 
puntos que va desde "muy alta" (5 puntos) a "muy baja" (1 punto). Adicional a ello se agregó una pregunta sobre satisfacción global del servicio recibido.

Esta primera versión fue presentada a "Juicio de expertos", conformada por un equipo multidisciplinario de profesionales especialistas en Nutrición, Gerencia de servicios de salud e Investigación. Cada experto evaluó los ítems a fin de cotejarlos de manera cualitativa con los criterios propuestos a relevancia o congruencia con los fines que persigue medir la encuesta, claridad en la redacción, tendenciosidad o sesgo en su formulación y dominio del contenido. Ellos mostraron observaciones a las preguntas 1,3, 5, 6, 7 y 13 a cerca del grado de dificultad que puedan tener los pacientes para interpretar los términos como "menaje, sabor, bebidas calientes, bebidas frías, preferencias alimentarias". Es por ello que se agregó ejemplos o sinónimos al lado de estos términos para facilitar su comprensión. Una vez levantadas las observaciones finalmente se aprobó en un 100\% los ítems.

\section{Prueba Piloto:}

Con la versión SERVNUT 2.0 se realizó una primera prueba durante el mes de diciembre del 2017 a 20 pacientes hospitalizados que cumplían los criterios de inclusión propuestos para esta investigación (Paciente hospitalizado cuya edad oscile entre 18 a 59 años, con indicación médica de dieta blanda que haya recibido por lo menos 3 comidas y que deseen participar del estudio). Previo conocimiento del objetivo de investigación las personas participaron de forma voluntaria. No se tuvo observaciones en cuanto a la interpretación de los términos de las preguntas. Por lo que finalmente el instrumento adaptado SERNUT 2.0, mantuvo 16 preguntas. 15 de ellas agrupadas en 5 dimensiones: Elementos tangibles contiene las preguntas del 1 al 3; Fiabilidad del 4 al 7; Capacidad de respuesta preguntas 8 y 9; Seguridad del 10 al 12; Empatía del 12 al 15. La pregunta 16 busca evaluar la satisfacción en general del paciente con respecto al Servicio de Nutrición. Las preguntas para caracterizar a los pacientes incluyeron datos de sexo, edad, grado de instrucción, ocupación, tiempo de estancia hospitalaria y apetito.

Esta última versión del instrumento se aplicó al tamaño de muestra definida mediante el método sistemático durante los meses de enero a mayo del 2018 al momento del alta de su hospitalización previo consentimiento de los mismos.

\section{Análisis de la validez del cuestionario:}

Para evaluar la validez de constructo, se utilizó la técnica del Análisis Factorial Exploratorio de Componentes Principales, luego con la finalidad de alcanzar una mejor comprensión de la matriz resultante se aplicó la rotación de Varimax. La medida de adecuación muestral de Kaiser-Meyer-Olkin (KMO), el Test de esfericidad de Barlett y el determinante de la matriz de correlaciones, fueron utilizados para comprobar los supuestos de aplicación. La fiabilidad se evaluó mediante el coeficiente Alfa de CronBach.

\section{RESULTADOS}

En la Tabla 1 se muestran las características generales y sociodemográficas de la muestra en estudio.

\section{Validez del constructo:}

Para la validación del instrumento, se efectuó un Análisis Factorial Exploratorio de Componentes Principales con los 15 ítems, previamente se verificó su viabilidad a través de las correlaciones bivariadas significativas, se obtuvo un índice $\mathrm{KMO}$ de 0.778 y una prueba de esfericidad de Barlett de 607.4 ( $p<0.000)$. La varianza explicada por los 5 factores obtenidos fue $69.712 \%$, cuyos autovalores se encontraron por encima de uno (Tabla 2).

En la Tabla 3, se muestra la asignación de los ítems a cada uno de los cuatro factores establecidos, el cual se hizo a través de las cargas factoriales, para lo cual se realizó una rotación Varimax de los factores, con el objetivo de redistribuir las cargas factoriales y facilitar su clasificación, sin afectar la proporción de la varianza explicada.

\section{Confiabilidad:}

Se analizó la fiabilidad evaluando la consistencia interna mediante el coeficiente Alfa de Cronbach, obteniéndose un valor de 0.848 Versión final del cuestionario SERVNUT 2.0.

\section{Servnut 3.0:}

En la Tabla 4 muestra la encuesta validada SERVPERF con sus 22 ítems organizados en 5 dimensiones mientras que en la Tabla 5 se observa la encuesta SERVNUT 3.0 propuesta que aparece a partir del análisis factorial. La encuesta contiene una nueva dimensión "Experiencia Gastronómica" la cual agrupa las dimensiones originales: Elementos tangibles, fiabilidad y seguridad. 
Tabla 1 . Características Generales.

\begin{tabular}{|c|c|}
\hline CARACTERISTICA & $\%$ \\
\hline \multicolumn{2}{|c|}{ Rango de edad } \\
\hline Promedio & {$[32.9 \pm 7.877]$} \\
\hline $18-29$ & 34 \\
\hline $30-39$ & 51 \\
\hline $40-49$ & 10 \\
\hline $50 a+$ & 5 \\
\hline \multicolumn{2}{|c|}{ Sexo } \\
\hline $\mathrm{F}$ & 94 \\
\hline$M$ & 6 \\
\hline \multicolumn{2}{|c|}{ Grado de Instrucción } \\
\hline Secundaria & 6 \\
\hline Técnico & 22 \\
\hline Superior & 72 \\
\hline \multicolumn{2}{|c|}{ Estancia Hospitalaria } \\
\hline$<1$ semana & 97 \\
\hline$>1$ semana & 3 \\
\hline
\end{tabular}

Tabla 2 . Análisis Factorial.

\begin{tabular}{ccccc}
\hline COMPONENTE & AUTOVALOR & \% DE VARIANZA & \% ACUMULADO & TOTAL \\
\hline 1 & 3.055 & 34.414 & 34.414 & 3.055 \\
\hline 2 & 2.348 & 13.505 & 47.919 & 2.348 \\
\hline 3 & 1.924 & 8.506 & 56.425 & 1.924 \\
\hline 4 & 1.752 & 6.754 & 63.178 & 1.752 \\
\hline 5 & 1.378 & 6.534 & 69.712 & 1.378 \\
\hline
\end{tabular}

Tabla 3 . Matriz de Componentes.

\begin{tabular}{|c|c|c|c|c|c|c|}
\hline \multirow{2}{*}{ No ÍTEM } & \multirow{2}{*}{ ÍTEM } & \multicolumn{5}{|c|}{ COMPONENTES } \\
\hline & & 1 & 2 & 3 & 4 & 5 \\
\hline 1 & Presentación de menaje & 618 & -.130 & -.372 & -.133 & -.218 \\
\hline 2 & Menaje limpio y desinfectado & .592 & .383 & -.304 & -.420 & -.041 \\
\hline 3 & Personal aseado & .666 & .386 & -.065 & -.274 & .139 \\
\hline 4 & Presentación del plato & .729 & -.489 & -.050 & .191 & -.021 \\
\hline 5 & Sabor & 648 & -.540 & .028 & .168 & .111 \\
\hline 6 & Temperatura platos calientes & .486 & -.343 & .075 & -.380 & .545 \\
\hline 7 & Temperatura platos fríos & .711 & -.106 & .240 & -.382 & -.014 \\
\hline 8 & Horario de atención & .549 & .096 & .556 & .010 & -.307 \\
\hline 9 & Prontitud en la atención & .373 & .302 & .689 & -.061 & -.175 \\
\hline 10 & Variedad & 617 & .022 & -.125 & .188 & -.397 \\
\hline 11 & Cantidad & .501 & -.297 & .066 & .233 & -.003 \\
\hline 12 & Dieta y estado de salud & .644 & -.336 & -.033 & .212 & .117 \\
\hline 13 & Preferencias alimentarias & .533 & .239 & -.410 & .026 & -.300 \\
\hline 14 & Trato del nutricionista & .487 & .566 & .014 & .391 & .402 \\
\hline 15 & Trato del personal & .536 & .612 & -.041 & .309 & .233 \\
\hline
\end{tabular}


Tabla 4 . Encuesta SERVPERF

DIMENSIONES

ÍTEMS

$\mathbf{N}^{\circ}$ ÍTEMS

La empresa debe tener equipos de aspectos modernos

Las instalaciones físicas de la empresa deben ser atractivas

Elementos

tangibles

Los empleados de la empresa deben verse pulcros

Los materiales asociados con el servicio deben ser visualmente atractivos para empresa de publicidad

\section{Cuando compromete hacer algo en cierto tiempo lo debe cumplir}

Cuando el cliente tiene un problema, la empresa debe mostrar un sincero interés en resolverlo

Fiabilidad

La empresa debe desempeñar bien el servicio por primera vez

La empresa debe proporcionar sus servicios en el momento en que promete hacerlo

La empresa debe insistir en registros libres de error

La empresa debe mantener informados a los clientes con respecto a cuándo se ejecutarán los servicios

Capacidad de

Los empleados deben dar un servicio rápido

respuesta

Los empleados deben estar dispuestos a ayudarles

Los empleados de la empresa, nunca deben estar desanimados u ocupados para ayudarles

El comportamiento de los empleados debe infundir confianza en usted

El cliente debe sentirse seguro de las transacciones con la empresa

Seguridad

Los empleados deben ser corteses de manera constante con usted

Los empleados de la empresa de publicidad, deben tener conocimiento para responder a las preguntas de los clientes

La empresa debe dar atención individualizada a los clientes

La empresa de publicidad debe tener empleados que den atención personal a cada uno de los clientes

Empatía

Los empleados deben entender las necesidades específicas de usted

La empresa de publicidad debe tener horarios de atención convenientes para la empresa de publicidad 
Tabla 5 . Encuesta propuesta SERVNUT 3.0.

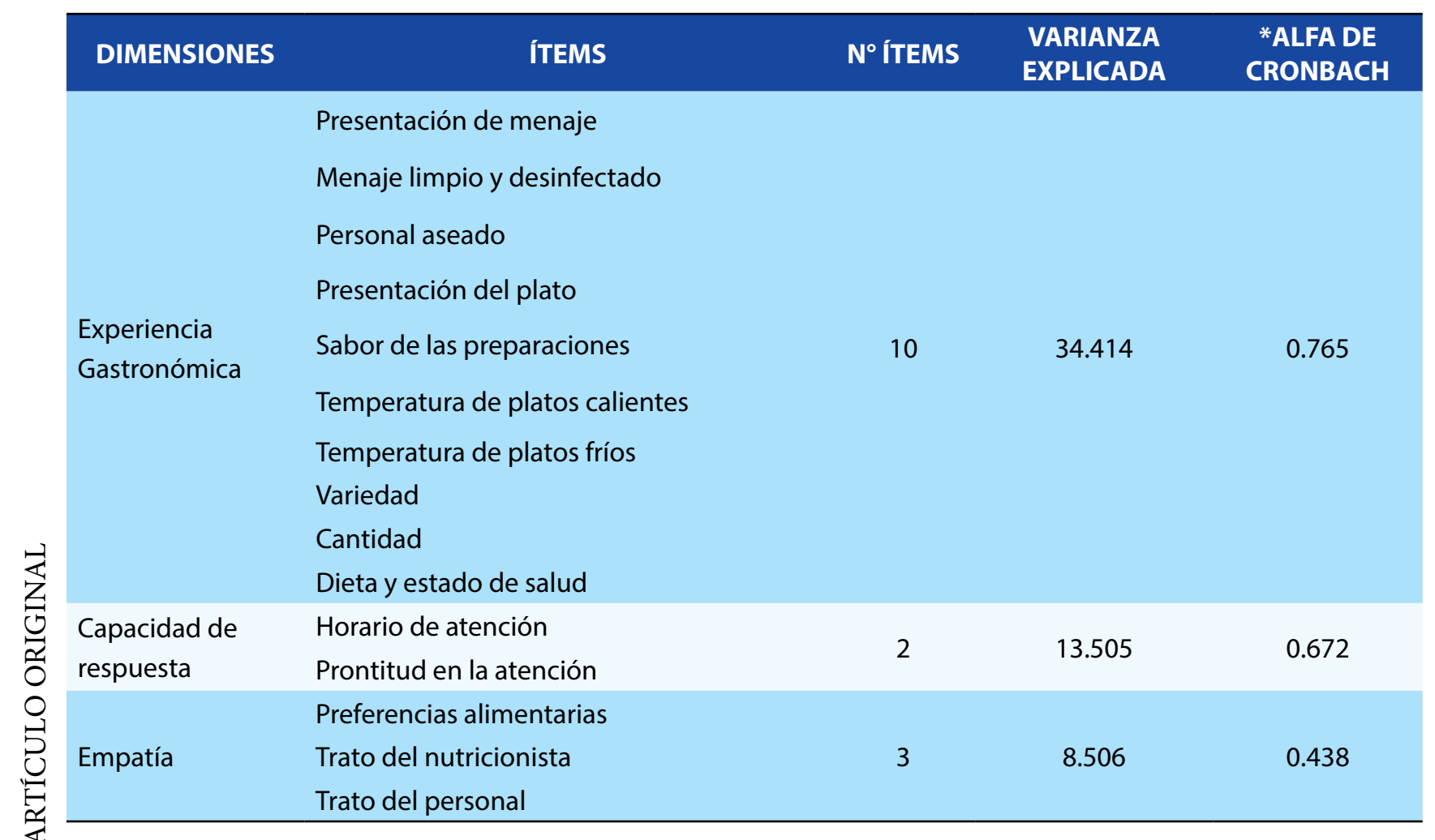

\section{DISCUSIÓN}

En la actualidad la importancia de la aplicación de encuestas a los usuarios de los Servicios de Salud con el fin de evaluar la calidad percibida de los mismos es bien conocida, debido a la practicidad de la metodología y a la confiabilidad de los resultados los cuales serán canalizados en toma de decisiones por parte de los gestores. Asimismo autores exponen que existe una la alta asociación entre la satisfacción del usuario y la calidad del servicio, lo que conlleva a prestar atención en la medición de este indicador. 18- 20 En este contexto realizamos la búsqueda de instrumentos validados para evaluar la satisfacción de los usuarios que reciben la atención de los Servicios de Nutrición durante su hospitalización, sin embargo no hallamos ningún resultado en nuestro medio. Teniendo en cuenta que la calidad de atención en los Servicios de Salud, tiene un carácter multidimensional los investigadores no se ponen de acuerdo respecto a la composición del constructo. Por este hecho se reporta en la literatura diferentes instrumentos para medir la calidad de atención con diferentes dimensiones, extensiones y contenidos; este es el caso de los Servicios de Nutrición el cual es multidimensional influenciado por una variedad de factores técnicos, ambientales e interpersonales, por ello decidimos construir una encuesta que sea sensible y fiable a partir del modelo SERVPERF ${ }^{21}$.
Para validar el contenido de nuestro instrumento se utilizó la técnica de Delphi, la cual al representar un juzgamiento colectivo sistematizado se hace relevante a diferencia de la opinión de un solo individuo ${ }^{22,23}$. Se considera que esta técnica no indica un determinante absoluto sobre los resultados por ello es necesario otros parámetros para confirmar la validez $z^{24,25}$.

Continuando con el estudio de la validez se utilizó el método estadístico de Análisis Factorial Exploratorio usando el método de Componentes Principales, la cual a diferencia del método de Factor Común el cual pretende entender la estructura de variables latentes que explican la relación entre las variables, el método de Componente Principal busca reducir el número de variables; la elección del método depende de los objetivos que persigue la investigación ${ }^{26-28}$.

Posterior al Análisis Factorial de Componentes Principales se realizó la prueba de Rotación Varimax para conocer en profundidad las variables más relacionadas con cada factor.

La prueba de Rotación Varimax se aplica con el supuesto que los factores no están correlacionados entre sí y por ende es adecuada al extraer factores a partir de componentes principales que permiten obtener dimensiones independientes. A diferencia del método de Rotación Oblicua que se utiliza cuando la matriz de correlaciones entre factores no es una matriz identidad ${ }^{29,30}$. 
Los resultados que muestra esta prueba estadística nos sugiere que las preguntas 1,2,3,4,5,6,7,10,11 pertenecientes a las dimensiones originales: "Elementos tangibles" la cual está relacionada con la presentación y el estado de los utensilios; "Fiabilidad" que se refiere a la calidad en sí de las preparaciones y de los insumos y "Seguridad" que se relaciona directamente con la calidad de las preparaciones; deberían formar parte de una sola dimensión a la cual denominamos "Experiencia gastronómica". Coincidentemente estas preguntas se encuentran relacionadas con la calidad de las preparaciones que se sirven a los pacientes. Mientras que las demás preguntas se cargaron correctamente en las dimensiones "Capacidad de respuesta" y "Empatía" las cuales van direccionadas con la atención que brinda el personal Servicio de Nutrición. De manera similar Babakus y Mangold ${ }^{31}$ en un estudio en EE.UU. sobre la adaptación de SERVQUAL a los servicios hospitalarios no logró identificar claramente las dimensiones de las expectativas y percepciones, que comprenden el modelo original de SERVQUAL (Elementos tangibles, fiabilidad, seguridad, capacidad de respuesta, empatía). Esto sugiere que los pacientes pueden percibir los servicios hospitalarios de manera diferente en comparación con los clientes de las demás empresas prestadoras de servicios. De otro lado a nivel internacional la literatura reporta una encuesta validada Acure Care Hospital Foodservice Patenta Satisfaction Questionnaire (ACHFPSQ). La encuesta ACHFPSQ es una herramienta utilizada en diversos estudios de Australia, Italia, España, etc. de esta índole ${ }^{17}$. Esta encuesta reúne información general similar a SERVNUT 3.0 acerca de la edad, sexo, tiempo de estancia, apetito, tipo de dieta y necesidad de ayuda para los pacientes al momento de ingerir sus alimentos. Comprende 16 preguntas sobre las dimensiones: calidad de los preparaciones, calidad del servicio, preguntas sobre el personal, servicio y entorno físico, más dos preguntas no incluidas en estas dimensiones y una sobre la satisfacción general. Dichos autores no sugieren el uso de dimensiones SERVQUAL sino variables asociados con la satisfacción de las comidas en hospitales que la literatura reporta ${ }^{32-35}$.

El instrumento final propuesto podría estar conformado por 15 ítems agrupadas en 3 Dimensiones: “Experiencia Gastronómica, Capacidad de respuesta, Empatía" pero esto necesita ser confirmado en futuros estudios, por lo que mantenemos la versión final con 5 dimensiones.

La fiabilidad de nuestro instrumento fue medido utilizando el coeficiente Alfa de Cronbach, la cual se ve relacionada con el grado de error aleatorio. En el sentido práctico una medición es fiable cuando suministra resultados constantes ya sea en mediciones repetidas o en las respuestas a los otros ítems que componen el instrumento ${ }^{36}$.

Las limitaciones de la presente investigación se encuentran en la homogeneidad de algunas características de la muestra como el sexo de los participantes. En este caso el $96 \%$ de los participantes eran de sexo femenino, lo cual puede ocasionar sesgos al momento de generalizar los resultados, esto se debió a que las características de la población objetivo a la cual va dirigido esta encuesta (Adultos de 18 a 59 años que reciban dieta blanda sin restricciones alimenticias que interfieran en la percepción de algunos componentes evaluados para medir la satisfacción de los Servicios de Nutrición), para el caso de la institución evaluada la población objetivo en su mayoría coincidió con pacientes de sexo femenino. Por ello es necesario aplicar este instrumento en otras instituciones de salud privada o pública para discriminar mejor los resultados ${ }^{37}$.

En cuanto al tamaño de la muestra autores refieren que este aspecto es una cuestión importante dado que afecta a la precisión de los resultados. Recientemente autores señalan que una muestra de 50 es un valor mínimo razonable, sin embargo es preferible trabajar con 100 o más unidades en la muestra ${ }^{38}$.

Finalmente presentamos la encuesta SERVNUT, la cual mediante las diferentes pruebas métricas presentadas ha demostrado reunir las características necesarias para ser considerado un instrumento útil y fiable para medir la satisfacción de los pacientes hospitalizados que reciben la atención del Servicio de Nutrición.

\section{CONCLUSIÓN}

El instrumento adaptado y validado SERVNUT posee propiedades psicométricas suficientes para ser considerado una herramienta útil y fiable para medir la satisfacción de pacientes hospitalizados con respecto al Servicio de Nutrición recibido durante su estadía en una institución privada de salud y con potencial aplicación en los diversos subsectores de salud del Perú. 
Código: Fecha:

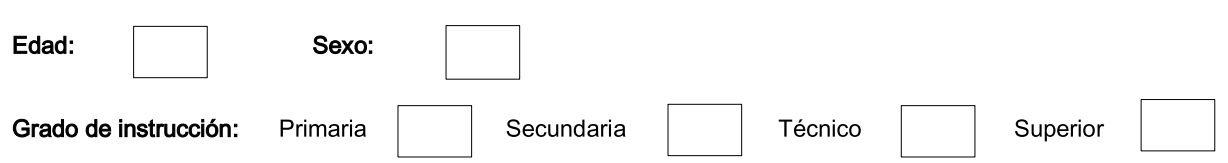

Ocupación:

Apetito: $\quad$ Normal $\square$ Bajo $\square$ Alto $\square$

Tiempo de estancia de hospitalización en la clínica: Menos de 1 semana

De 1 a 2 semanas

Estimado (a), estamos interesados en conocer su opinión sobre la calidad de atención que recibió del Departamento de Nutrición y Dietética. Sus respuestas son totalmente confidenciales.

Por favor leer los enunciados y marcar una opción: Considere 1 como la menor calificación y 5 la mayor calificación.

$$
\begin{array}{|l|l|l|l|l|}
\hline \text { I=Muy baja } & 2=\text { Baja } & 3=\text { Media } & 4=\text { Alta } & 5=\text { Muy alta } \\
\hline
\end{array}
$$

\begin{tabular}{|c|c|c|c|c|c|c|}
\hline 1 & $\begin{array}{l}\text { ¿La presentación del menaje (plato, vaso, cubierto, bandeja) fue } \\
\text { atractiva? }\end{array}$ & 1 & 2 & 3 & 4 & 5 \\
\hline 2 & ¿El menaje estuvo limpio y en buen estado? & 1 & 2 & 3 & 4 & 5 \\
\hline 3 & ¿El personal que le entrega la dieta luce aseado (limpio, arreglado)? & 1 & 2 & 3 & 4 & 5 \\
\hline 4 & $\begin{array}{l}\text { ¿La presentación, que se refiere a la ubicación de los elementos del } \\
\text { plato, los colores o la decoración; hicieron visualmente agradable a } \\
\text { la comida? }\end{array}$ & 1 & 2 & 3 & 4 & 5 \\
\hline 5 & $\begin{array}{l}\text { ¿El sabor (dulce, salado, ácido, amargo) de las comidas fue } \\
\text { agradable? }\end{array}$ & 1 & 2 & 3 & 4 & 5 \\
\hline 6 & $\begin{array}{l}\text { ¿La temperatura de los platos y bebidas calientes (sopa, fondo, } \\
\text { cereal cocido, infusión, etc.) estuvieron deseables? }\end{array}$ & 1 & 2 & 3 & 4 & 5 \\
\hline 7 & $\begin{array}{l}\text { ¿La temperatura de los platos y bebidas frías (ensaladas, postres, } \\
\text { etc.) estuvieron deseables al paladar? }\end{array}$ & 1 & 2 & 3 & 4 & 5 \\
\hline 8 & ¿El horario de entrega de las comidas fue oportuna? & 1 & 2 & 3 & 4 & 5 \\
\hline 9 & $\begin{array}{l}\text { ¿Se le atendió con prontitud ante sus necesidades y preocupaciones } \\
\text { sobre su alimentación? }\end{array}$ & 1 & 2 & 3 & 4 & 5 \\
\hline 10 & $\begin{array}{l}\text { ¿Los menús programados fueron variados es decir que no se } \\
\text { repitieron los menús durante mi estancia hospitalaria? }\end{array}$ & 1 & 2 & 3 & 4 & 5 \\
\hline 11 & ¿La cantidad de las comidas fue la adecuada para mí? & 1 & 2 & 3 & 4 & 5 \\
\hline 12 & ¿La dieta que recibí me ayudó a mejorar mi salud? & 1 & 2 & 3 & 4 & 5 \\
\hline 13 & $\begin{array}{l}\text { ¿El nutricionista tomó en cuenta mis preferencias alimentarias en } \\
\text { mi dieta? Ejemplo: No consumo leche porque me cae mal pero me } \\
\text { entregaron yogurt y queso para remplazarlo. }\end{array}$ & 1 & 2 & 3 & 4 & 5 \\
\hline 14 & ¿El trato del nutricionista fue amable? & 1 & 2 & 3 & 4 & 5 \\
\hline 15 & ¿El trato del personal que entregó la comida fue amable? & 1 & 2 & 3 & 4 & 5 \\
\hline
\end{tabular}

En general: ¿Cuál es su grado de satisfacción con respecto a la alimentación que le ha proporcionado la clínica Good Hope?

\begin{tabular}{|l|l|l|l|l|}
\hline $1=$ Muy baja & $2=$ Baja & $3=$ Normal & $4=$ Alta & $5=$ Muy alta \\
\hline
\end{tabular}

¡GRACIAS POR SU COLABORACIÓN!

Figura 1 . Anexo, encuesta satisfacción. 
Contribuciones de autoría: Los autores participaron en la generación, recolección de información, redacción y aprobación final del artículo original.

Financiamiento: Autofinanciado.

Conflicto de interés: Los autores declaran no tener conflicto de interés en la publicación de este artículo.

Recibido: 02 de setiembre del 2018

Aprobado: 08 de octubre del 2018

\section{Correspondencia: Elizabeth Rojas-Apolinario}

Dirección: INICIB, Facultad de Medicina Humana, Edificio I-208. 2do piso. Avenida Benavides 5440, Surco, Lima-Perú.

Teléfono: 966416379

Correo:mili_era03@hotmail.com

\section{REFERENCIAS BIBLIOGRÁFICAS}

1. Guru, C. (2003). Tailoring e-service quality through CRM. Managing Service Quality, 13 (6), 520- 531

2. ISO 9001: 2000 - Sistemas de Gestión de Calidad. Requisitos [Internet].www.chospab.es/calidad/archivos/Documentos/ NormalnternacionallSO9001.pdf

3. EFQM. Directrices para el sector público: salud. Madrid: Club Gestión de Calidad. 1996.

4. Clemente P, Fortuna B, Tort-Martorell X. Modelo de excelencia de EFQM aplicado al ámbito sanitario. Barcelona: Faura Casas Editorial. 2003.

5. Joint Commission International Accreditation Standards for Hospitals, 6th Edition

6. Donabedian A. Evaluating the quality of medical care. The Milbank memorial fund quarterly. 1966; 44 (3):166-206.

7. Ware JE Jr, Davies-Avery A, Stewart AL. The measurement and meaning of patient satisfaction. Health Med Care Service Review; 1(1):13-15. 197

8. Crow $\mathrm{R}$, Gage $\mathrm{H}$, Hampson $\mathrm{S}$ et al. The measurement of satisfaction with healthcare: implications for practise from a systematic review of the literature. Health Technol Assess. 2002; 6:1-245.

9. Parasuraman A, ZeithamI VA, Berry LL. SERVQUAL: A multi-item scale measuring consumer perceptions of service quality. Journal of Retailing 1988; 64(1): 12-37

10. Cronin JJ, Jr., Taylor SA. Measuring Service Quality: A Reexamination and Extension. Journal of Marketing. 1992; 56(3):55-68.

11. Cronin Jr JJ, Taylor SA. SERVPERF versus SERVQUAL: Reconciling performance-based and perceptions-minus-expectations measurement of service quality. The Journal of Marketing. 1994; 125-131.

12. GregoireM.Quality of patient meal service in hospitals: delivery of meals by dietary employees vs delivery by nursing employees. J Am Diet Assoc 1994; 94: 1129-34.

13. Wright O, Capra S, Aliakbari J. A comparison of two measures of hospital foodservice satisfaction. Aust Health Rev 2003; 26: 70-5.

14. Askew D, Capra S, Sardie M. New perspectives in measuring client satisfaction with foodservice. Centre for Public Health Research Best Practice in Nutrition and Dietetic Research. Brisbane: Queensland University of Technology, 1996.

15. Wright ORL, Connelly LB, Capra S. Consumer evaluation of hospital foodservice quality: an empirical investigation. Int J Health Care Qual Assur 2006; 19: 181-94

16. Pinzón Espitia, O. L., Pardo Oviedo, J. M., \& González Rodríguez, J. L. (2015). Modelo Mederi de gestíon nutricional hospitalaria. Nutr Hosp 32(4), 1802-1807

17. Capra S, Wright O, Sardie M et al. (2005) The Acute Hospital Foodservice Patient Satisfaction Questionnaire: the development of a valid and reliable tool to measure patient satisfaction with acute care hospital foodservice. Foodservice Res Int 16, 1-14.

18. Jabrí RI. Patient healing vs customer satisfaction. Otolaryngol Head Merck Surg 2006; 134(4): 715.

19. kinney WC. A simple and valuable approach for measuring customer satisfaction. Otolaryngol Head Neck Surg 2005; 133(2): 169-7.

20. Wright JTC, Giovinazzo RAD. Delphi: Uma ferramenta de apoio ao planejamento prospectivo. Cad Pesqui Adm. 2016; 1(12):54-65.
21. McDougall GH, Levesque TJ. A revised view of service quality dimensions: An empirical investigation. Journal of Professional Service Marketing. 1994; 11(1): 189-209.

22. Munaretto LF, Corrêa HL, Cunha JAC. Um estudo sobre as características do método Delphi. Rev. Latino-Am. Enfermagem 2016.

23. Minayo MCS. Construção de indicadores qualitativos para avaliação de mudanças. Rev Bas Educ Méd. 2009.

24. Teixeira M, Faria SA, Lima B, Souza R, Eliete A, Azevedo G. Validación de un instrumento para evaluación de servicios de atención a la salud de la persona ostomizada. 1Rev. Latino-Am. Enfermagem 2016; 24: 2825. 25. Conway, J, Huffcutt, A. A review and evaluation of exploratory factor analysis practices in organizational research. Organizational Research Methods. 2003; 6(2), 147-168.

26. Costello A, Osborne J. Best practices in exploratory factor analysis: Four recommendations for getting the most from your analysis. Practical Assessment, Research \& Evaluation, 2005; 10(7).

27. Irini M. Análisis factorial exploratorio: cuestiones conceptuales y metodológicas. Nebrija De Lingüística Aplicada a la Enseñanza de las lenguas. 2015; 19.

28. Lloret S. Adoración Ferreres-Traver, Hernández B, Tomás M. E Análisis Factorial Exploratorio de los ítems: Una guía práctica, revisada y actualizada. anales de psicología. 2014; 30(3) 1151-1169.

29. Dolores F, Pascual S. Prácticas del Análisis Factorial Exploratorio en la Investigación sobre Conducta del consumidor y Marketing. Suma Psicológica. 2012; 19(1)47-58.

30. Batista F, Coenders G. Introducción a los modelos estructurales. Utilización del análisis factorial confirmatorio para la depuración de un cuestionario. En: Renom J, editor. Tratamiento informatizado de datos. Barcelona: Masson, 1998; p. 22.

31. BABAKUS, E. and MANGOLD, G. 1991. Adapting the SERVQUAL scale to hospital services: An empirical investigation. Health Serv. Res. 26, 767786

32. GREGOIRE, M.B. 1994. Quality of patient meal service in hospitals: Delivery of meals by dietary employees vs delivery by nursing employees. J. Am. Diet. Assoc. 94, 1129-1134.

33. O'HARA, P.A, HARPER, D.W KANGAS, M, DUBEAU, נ, BORSUTZK, C. and LEMIRE, N. 1997. Taste, temperature and presentation predict satisfaction with foodservices in a Canadian continuing-care hospital. J. Am. Diet. Assoc. 97, 401-405.

34. DUBE, L., TRUDEAU, E. and BELANGER, M.C. 1994. Determining the complexity of patient satisfaction with food services. J. Am. Diet. Assoc. 94, 394-398.

35. LAU, C. and GREGOIRE, M.B. 1998. Quality ratings of a hospital foodservice department by inpatients and postdischarge patients. J. Am. Diet. Assoc. 98, 1303-1307.

36. Frías N, Pascual S. Suma Psicológica. 2012; 19(1) 47-58.

37. Hair J, Anderson R, Tatham R, Black W. Análisis Multivariante. Madrid: Pearson. 2004

38. Cattell, R.B. The scientific use of factor analysis in behavioral and rifé sciences. New York, NY: Plenum Press. 1978. 